\title{
Relativistic Oscillators in a Noncommutative Space: a Path Integral Approach
}

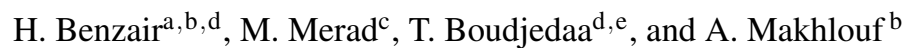 \\ ${ }^{a}$ Laboratoire LRPPS, Université de Kasdi Merbah-Ouargla, BP 511, Route Ghardaïa, 30000 \\ Ouargla, Algérie (permanent address) \\ b Laboratoire de Mathématiques, Informatique et Applications, Université de Haute-Alsace, 4 \\ rue des Frères Lumière F-68093 Mulhouse, France \\ ${ }^{c}$ Laboratoire (L.S.D.C), Département des Sciences de la Matière, Faculté des Sciences Exactes \\ et Sciences de la Vie, Université de Oum El Bouaghi, 04000 Oum El Bouaghi, Algérie \\ ${ }^{\mathrm{d}}$ Laboratoire de Physique Théorique, Université de Jijel BP98 Ouled Aissa, 18000 Jijel, Algérie \\ (permanent address) \\ e Laboratoire de Physique Théorique, Université Paris-Sud 11, Bâtiment 210, Orsay Cedex \\ France (visitor address)
}

Reprint requests to M. M.; E-mail: meradm@gmail.com

Z. Naturforsch. 67a, 77 - 88 (2012) / DOI: 10.5560/ZNA.2011-0060

Received January 11, 2011 / revised October 3, 2011

In this paper, we consider the dynamics of Klein-Gordon and Dirac oscillators in $(2+1)$ dimensions with noncommutativity of the spatial coordinates using the supersymmetric path integral formalism. The propagator is calculated and the energy eigenvalues with their corresponding eigenfunctions are deduced.

Key words: Noncommutative Geometry; Path Integral; Klein-Gordon and Dirac Oscillators. PACS numbers: 03.65.Pm; 03.65.Ca; 03.65.Db; 03.65.Ge

\section{Introduction}

Recently, the noncommutative (NC) geometry received a great welcome by the researchers in the field of physics and mathematics. Its roots lie in quantum mechanics, describing at microscopic level the laws of nature. Quantum mechanics motivated also in the first half of the twentieth century an important development in the theory of operator algebras, like the study of $C^{*}$-algebra and Von Neumann algebras. We know that from classical mechanics to quantum mechanics, one changes the commutative algebra of functions on the phase space to a noncommutative operator algebra on a Hilbert space. A similar procedure can be realized in geometry where the classical notions loose their applicability and pertinence and can be replaced by a new idea of space, represented by noncommutative algebras $[1,2]$.

The noncommutative space representation can be realized by the coordinate operators $\tilde{x}^{\mu}, \mu=0,1,2$, sat- isfying the commutation relations (throughout the paper we adopt the natural units $\hbar=c=1)$ :

$$
\left[\tilde{x}^{\mu}, \tilde{x}^{v}\right]=\mathrm{i} \theta^{\mu v}
$$

where $\theta^{\mu v}$ is a $(2+1)$-dimensional anti-symmetric matrix with constant elements, where the antisymmetric matrix can be simply chosen as $\theta^{\mu v}=\theta \varepsilon^{\mu v}$ and $\theta^{\mu 0}=\theta^{0 \mu}=0$, where $\varepsilon^{\mu v}$ is the Levi-Civita symbol and $\theta$ is a parameter that measures the noncommutativity of coordinates, (see, e.g., [3] for a review on noncommutative geometry). The framework of Weyl's quantization procedure [4] provides a formalism that associates to the algebra of noncommuting coordinates $(\hat{A}, \bullet)$ an algebra of functions of commuting variables with deformed product $(A, *)$. We define a map $W: A \rightarrow \hat{A}$ such that an element from $\hat{A}$ is assigned to a function $f\left(x_{0}, x_{1}, x_{2}\right)=f(x)$ from $A$

$$
W(f)=\hat{f}=\frac{1}{(2 \pi)^{\frac{3}{2}}} \int \mathrm{d}^{3} k \mathrm{e}^{\mathrm{i} k_{v} \tilde{x}} \tilde{f}(k),
$$


where $\tilde{f}(k)$ is the Fourier transform of $f(x)$,

$$
\tilde{f}(k)=\frac{1}{(2 \pi)^{\frac{3}{2}}} \int \mathrm{d}^{3} x \mathrm{e}^{-\mathrm{i} k_{v} x^{v}} f(x) .
$$

The multiplication of two operators $W(f)$ and $W(g)$ obtained from (2) yields another operator $W(f * g)$ :

$$
W(f) \bullet W(g)=\hat{f} \bullet \hat{g}=W(f * g)
$$

with $f * g \in(A, *)$, a classical function which is well defined, as shown in sequel. Substituting (2) in (4) we obtain:

$$
\begin{aligned}
W(f * g) & =W(f) \bullet W(g) \\
& =\frac{1}{(2 \pi)^{3}} \int \mathrm{d}^{3} k \mathrm{~d}^{3} p \mathrm{e}^{\mathrm{i} k_{\mu} \tilde{x}^{\mu}} \mathrm{e}^{\mathrm{i} p_{v} \tilde{x}^{\nu}} \tilde{f}(k) \tilde{g}(p) .
\end{aligned}
$$

In the case of canonical noncommutativity as given by (1), the product of the two exponentials in the above formula will give an exponential of a linear combination of the $\tilde{x}_{\mu}$ after applying the Baker-CampbellHausdorff formula

$$
\mathrm{e}^{\hat{A}} \mathrm{e}^{\hat{B}}=\mathrm{e}^{\hat{A}+\hat{B}+\frac{1}{2}[\hat{A}, \hat{B}]+\frac{1}{12}([\hat{A},[\hat{A}, \hat{B}]]+[[\hat{A}, \hat{B}], \hat{B}]+\ldots)}
$$

and considering the commutator relation $\left[\left[\tilde{x}^{\mu}\right.\right.$, $\left.\left.\tilde{x}^{v}\right], \tilde{x}^{\rho}\right]=0$ thus makes all terms including more than one commutator in (6) vanish:

$$
\mathrm{e}^{\mathrm{i} k_{\mu} \tilde{x}^{\mu}} \mathrm{e}^{\mathrm{i} p_{v} \tilde{x}^{v}}=\mathrm{e}^{\mathrm{i}\left(k_{v}+p_{v}\right) \tilde{x}^{v}-\frac{\mathrm{i}}{2} k_{\mu} p_{v} \theta^{\mu v}} .
$$

We obtain $f * g$ by comparing (5) with (2) and replacing the operator $\tilde{x}$ by the coordinate $x$ :

$$
\begin{aligned}
f * g= & \frac{1}{(2 \pi)^{3}} \int \mathrm{d}^{3} k \mathrm{~d}^{3} p \mathrm{e}^{\mathrm{i}\left(k_{v}+p_{v}\right) \tilde{x}^{\nu}-\frac{\mathrm{i}}{2} k_{\mu} p_{\nu} \theta^{\mu \nu}} \\
& \cdot \tilde{f}(k) \tilde{g}(p) .
\end{aligned}
$$

Thus, the Moyal-Weyl $*$-product is defined by:

$$
f(x) * g(x)=\exp \left[\frac{\mathrm{i}}{2} \theta^{\mu v} \partial_{x_{\mu}} \partial_{y_{v}}\right] f(x) g(y)_{\left.\right|_{x=y}},
$$

where $\partial_{x_{\mu}}$ is the partial derivative operator. Let us show that the star product inducing the noncommutativity is replaced by the usual product plus a nonlocal correction in the scalar function $f(x)$. Indeed, it is easy to show that

$$
\begin{aligned}
f(x) * g(x)= & f(x) \cdot g(x)+\sum_{n=1}^{\infty} \frac{1}{n !}\left(\frac{\mathrm{i}}{2}\right)^{n} \partial_{\mu_{1}} \ldots \partial_{\mu_{n}} f(x) \\
& \cdot \theta^{\mu_{1} v_{1}} \ldots \theta^{\mu_{n} v_{n}} \partial_{v_{1}} \ldots \partial_{v_{n}} g(x) .
\end{aligned}
$$

Now, we replace $\partial_{j_{k}}$ by i $p_{j_{k}}=\frac{\partial}{\partial x^{j_{k}}}$ and introduce $\mathcal{P}_{i_{k}}=$ $\theta^{i_{k} j_{k}} p_{j_{k}}$. We take the Fourier transform of $f(x)$, then

$$
\begin{aligned}
& \partial_{\mu_{1}} \ldots \partial_{\mu_{n}} f(x) \mathcal{P}_{\mu_{1}} \ldots \mathcal{P}_{\mu_{n}} g(x) \\
& =\mathrm{i}^{n} \int \mathrm{d}^{3} k \mathrm{e}^{\mathrm{i} k x} f(k)(k \mathcal{P})^{n} g(x) .
\end{aligned}
$$

Summing over $n$ in (10), we get:

$$
f(x) * g(x)=\int \mathrm{d}^{3} k \mathrm{e}^{\mathrm{i} k x} \mathrm{e}^{\frac{\mathrm{i}}{2} \mathcal{P} k} f(k) g(x) .
$$

Now using $\left[p_{i}, x_{j}\right]=-\mathrm{i} \delta_{i j}$, we obtain

$$
f(x) * g(x)=f\left(x-\frac{\mathcal{P}}{2}\right) \cdot g(x) .
$$

This result (13) is a crossing point from the noncommutative case to the commutative case, (i.e. the *-product may be changed into an ordinary product by shifting $\tilde{x}$ by $\left.x-\frac{\mathcal{P}}{2}\right)$.

One of the greatest successes of noncommutative geometry has been the unification of the forces of nature into a single gravitational action [5,6]. Furthermore, the noncommutative geometry plays an important role in string theory and M-theory [7-9]. In addition, the noncommutative spaces have been included in quantum field theory by a great number of scientific researchers, see for example [10-17]. At the same time, we find less interest in studying these issues from the Feynman point of view. The main difficulty for applying this formalism (Feynman technique) is in the discrete nature of the spin, as discussed in many papers [18-21]. The first treatment was given by Fradkin and Berzin based on the idea of replacing Dirac-Pauli matrices by the magic number (Grassmann variables $\psi^{\mu}$ ). Their fundamental idea is to write the formal equation of the causal Green function like the inverse of an operator and express this inverse as a standard evolution operator by using an integral representation. Generally, in the Dirac oscillator equation, we find a supersymmetric proper time having two parts: one fermionic and the other bosonic. In our work, we focus on the bosonic time, whose treatment is analogous to the so-called global projection [22]. This formalism was used in the noncommutative and commutative space-time in a few applications of quantum field theory [23-28].

The aim of this paper is to use supersymmetry formalism to calculate the Green function for the twodimensional relativistic Dirac and Klein-Gordon oscillators in a noncommutative space with and without the presence of a constant magnetic field $\mathcal{B}$ perpendicular 
to the noncommutative plane $(\tilde{x}, \tilde{y})$. Our results will be compared to those obtained by the Schrödinger formalism as determined by [29].

Instead of solving the path integral representation for the two-dimensional Dirac and Klein-Gordon oscillators in a noncommutative space by using the starproduct procedure, we use Bopp's shift [30], which is defined in (13). It is known that the nonrelativistic harmonic oscillator in a noncommutative space has a similar behaviour to the Landau problem in a commutative space [31]. We generalize this relation to relativistic quantum mechanics and state that the Dirac and KleinGordon oscillators in noncommutative space has a similar behaviour to the Dirac and Klein-Gordon oscillators in commutative space with the presence of a constant magnetic field. However, for the Dirac oscillator noncommutative case, a new term will appear which implies that a charged fermion in a noncommutative space has an electric dipole moment.

This paper is outlined as follows. In Section 2, we show, via the path integral representation, an explicit calculation of the Klein-Gordon oscillator in noncommutative space relative to a 0 -spin particle. The exact solution is obtained and the wave functions are expressed in terms of generalized Laguerre polynomials, as well as the energy spectra. In Section 3, and following the same steps, we treat the spin $1 / 2$ case in a noncommutative space with the help of the Fradkin and Gitman technique [23]. In addition, we also find some information on this physical system in the presence of a constant magnetic field just by replacing $\omega$ by $\varpi=\omega+\frac{e \mathcal{B}}{2 m}$, and we could also take the commutative space case when $\theta \rightarrow 0$. We obtain similar results than previous studies. A conclusion is given in Section 4 . We provide at the end of the paper an appendix reviewing the Grassmann integration technique.

\section{The Green Function of a Klein-Gordon Oscillator in a Noncommutative Space Representation}

Let $G(x, y)$ be the Green function of the usual Klein-Gordon oscillator for a spinless particle, then the propagator of the scalar particle on NC space is the causal Green function $G^{(\theta)}(x, y)$ of the Klein-Gordon oscillator equation. It is usually written as

$$
\begin{aligned}
& {\left[p_{0}^{2}+\left(p_{i}+\mathrm{i} m \omega x_{i}\right)\left(p^{i}-\mathrm{i} m \omega x^{i}\right)-m^{2}\right] * G(x, y)} \\
& \quad=-\delta^{3}(x-y),
\end{aligned}
$$

where the Moyal-Weyl (or star) product between the two functions (14) is defined in (8). We have shown in the preceding section that on NC quantum mechanics the Moyal *-product can be replaced by a Bopp's shift [30], and the operators terms $\hat{p}_{0}^{2}$ and $\hat{p}^{2}$ are unchanged, however the scalar function of $x$ will change, yielding the equivalent in a commutative space

$$
\begin{aligned}
& {\left[\hat{p}_{0}^{2}+\left(\widehat{\tilde{p}}_{i}+\mathrm{i} m \omega \widehat{\tilde{x}}_{i}\right)\left(\widehat{\tilde{p}}^{i}-\mathrm{i} m \omega \widehat{\tilde{x}}^{i}\right)-m^{2}\right] G^{(\theta)}\left(x_{f}, x_{i}\right)} \\
& \quad=-\delta^{3}\left(x_{f}-x_{i}\right) .
\end{aligned}
$$

The operators $\widehat{\tilde{x}}_{i}$ are noncommutative variables obeying the commutation relation (1). We can obtain (1) by using the following linear transformation between noncommutative variables $\left(\widehat{\tilde{x}}_{i}, \widehat{\tilde{p}}_{i}\right)$ and commutative variables $\left(\hat{x}_{i}, \hat{p}_{i}\right)$ :

$$
\begin{aligned}
& \widehat{\tilde{x}}_{i}=\hat{x}_{i}-\frac{\theta_{i j}}{2} \hat{p}_{j}, \text { with } i, j=1,2, \\
& \widehat{\tilde{p}}_{i}=\hat{p}_{i}
\end{aligned}
$$

where $\hat{p}_{i}$ are momentum operators conjugated to $\hat{x}_{i}$, which satisfy ordinary Heisenberg commutation. It appears therefore that the dynamics of a Klein-Gordon oscillator in a noncommutative space has similar behaviour to the same dynamics of a particle in a commutative space and in a constant magnetic field.

Following Schwinger [32], we present $G^{(\theta)}(x, y)$ as a matrix element of an operator $\hat{G}^{(\theta)}$,

$$
G^{(\theta)}\left(x_{f} ; y_{f}, x_{i} ; y_{i}\right)=\left\langle x_{f} y_{f}\left|\hat{G}^{(\theta)}\right| x_{i} y_{i}\right\rangle,
$$

Here $|x\rangle$ are eigenvectors of some self-adjoint operators of coordinates $\hat{x}_{i}$. The corresponding canonicalconjugated operators of momenta are $\hat{p}_{i}$ so that

$$
\begin{aligned}
& {\left[\hat{x}_{i}, \hat{p}_{j}\right]=\mathrm{i} \delta_{i j}, \hat{x}_{i}|x\rangle=x_{i}|x\rangle} \\
& \left\langle x \mid x^{\prime}\right\rangle=\delta^{3}\left(x-x^{\prime}\right) ; \int|x\rangle\langle x| \mathrm{d} x=I \\
& \hat{p}_{i}|p\rangle=p_{i}|p\rangle,\left\langle p \mid p^{\prime}\right\rangle=\delta^{3}\left(p-p^{\prime}\right) \\
& \int|p\rangle\langle p| \mathrm{d} p=I ;\langle x \mid p\rangle=\frac{1}{(2 \pi)^{3 / 2}} \mathrm{e}^{\mathrm{i} p x}
\end{aligned}
$$

Now one can use the Schwinger proper-time representation for the inverse operator. We get

$$
\begin{aligned}
& G^{(\theta)}\left(x_{f} ; y_{f}, x_{i} ; y_{i}\right) \\
& =-\mathrm{i} \int_{0}^{\infty}\left\langle x_{i} y_{i}\left|\exp \left(-\mathrm{i} \lambda\left(\hat{H}^{(\theta)}-\mathrm{i} \varepsilon\right)\right)\right| x_{f} y_{f}\right\rangle \mathrm{d} \lambda,
\end{aligned}
$$


where $\lambda$ is the proper-time and the infinitesimal $\varepsilon$ has to be sent to zero at the end of the calculations in order to reobtain the Green function (17). The Hamiltonian $\hat{H}^{(\theta)}$ consists of two terms: the first is the Hamiltonian operator of the usual quantum system and the other term depends on the noncommutative space $\theta$ :

$\hat{H}^{(\theta)}=\hat{p}_{0}^{2}-m^{2}-\left(\hat{p}_{x}^{2}+\hat{p}_{y}^{2}\right)-(m \omega)^{2}\left(\hat{x}^{2}+\hat{y}^{2}\right)+2 m \omega$

$-\frac{(m \omega \theta)^{2}}{4}\left(\hat{p}_{x}^{2}+\hat{p}_{y}^{2}\right)+(m \omega)^{2} \theta\left(\hat{x} \hat{p}_{y}-\hat{y} \hat{p}_{x}\right)$.

Here and in what follows, we include the factor $(-\mathrm{i} \varepsilon)$ in $m^{2}$. In order to derive a path integral representation for $G^{(\theta)}$, we follow the standard discretization method for the kernel (19) as done in [23]. Then we get the Lagrangian path integral representation for the Green function $G^{(\theta)}$,

$$
\begin{aligned}
& G^{(\theta)}\left(x_{f}, y_{f}, x_{i}, y_{i}, t_{f}, t_{i}\right) \\
& =-\mathrm{i} \int_{0}^{\infty} \frac{\mathrm{d} e_{0}}{2} \int D e D \pi_{e} \int D x D y D t \int D p_{0} \mathcal{M}^{(\theta)}(e) \\
& \cdot \exp \left\{\mathrm { i } \int _ { 0 } ^ { \frac { e } { 2 } } \left[p_{0}^{2}-m^{2}+\frac{\dot{x}^{2}+\dot{y}^{2}}{4 \omega_{1}}\right.\right. \\
& -(m \omega)^{2}\left(1-\frac{(m \omega)^{2} \theta^{2}}{4 \omega_{1}}\right)\left(x^{2}+y^{2}\right) \\
& \left.\left.+\frac{(m \omega)^{2}}{2 \omega_{1}} \theta(\dot{y} x-\dot{x} y)+2 m \omega+p_{0} \dot{t}+\pi_{e} \dot{e}\right] \mathrm{~d} s\right\}
\end{aligned}
$$

where $e(s)=2 \lambda(s)$ and $\omega_{1}=1+\frac{(m \omega \theta)^{2}}{4}$. The functional integration in (21) goes over trajectories $x_{\mu}(s)$, $p_{\mu}(s), e(s)$, and $\pi_{e}(s)$, parameterized by some invariant parameter $s \in[0,1]$ and obeying the boundary conditions $\vec{x}(0)=\vec{x}_{i}, \vec{x}(1)=\vec{x}_{f}, t(0)=t_{i}, t(1)=t_{f}$, $e(0)=e_{0}$; the measure $\mathcal{M}^{(\theta)}(e)$ has the form

$$
\begin{aligned}
& \mathcal{M}^{(\theta)}(e)=\int D p_{x} D p_{y} \\
& \cdot \exp \left\{-\mathrm{i} \int_{0}^{\frac{e}{2}}\left(1+\frac{(m \omega \theta)^{2}}{4}\right)\left(p_{x}^{2}+p_{y}^{2}\right) \mathrm{d} s\right\} .
\end{aligned}
$$

We can remove the functional integration over $t$ and $e$ after integrating over $p_{0}$ and $\pi_{e}$ which gives $\delta$ functions for $\dot{e}$ and $\dot{t}$. It is clear that this problem will be solved easily by the polar coordinates; then, the ex- pression of the Green function (21) becomes

$$
\begin{aligned}
& G^{(\theta)}\left(r_{f}, \varphi_{f}, r_{i}, \varphi_{i}, T\right)=\mathrm{i} \int_{0}^{\infty} \frac{\mathrm{d} e_{0}}{2} \int \frac{\mathrm{d} p_{0}}{2 \pi} \mathrm{e}^{-\mathrm{i} p_{0} T} \\
& \cdot \mathcal{M}\left(e_{0}, \omega_{1}\right) \exp \left\{\frac{\mathrm{i} e_{0}}{2}\left[p_{0}^{2}-m^{2}+2 m \omega\right]\right\} \\
& \cdot \int r D r(t) D \varphi(t) \exp \left\{\frac { \mathrm { i } } { 2 } \int _ { 0 } ^ { e _ { 0 } \omega _ { 1 } } \left[\left[\dot{r}^{2}\right.\right.\right. \\
& +r^{2} \dot{\varphi}^{2}-\frac{(m \omega)^{2}}{\omega_{1}}\left(1-\frac{(m \omega)^{2} \theta^{2}}{4 \omega_{1}}\right) r^{2} \\
& \left.\left.+\frac{(m \omega)^{2} \theta}{\omega_{1}} r^{2} \dot{\varphi}\right] \mathrm{d} s\right\} .
\end{aligned}
$$

After a shift on the angle $\varphi(s) \rightarrow \varphi(s)+\frac{(m \omega)^{2} \theta}{2 \omega_{1}} s$, this Green function (23) becomes formally identical with that of the radial path integral solution for the radial harmonic oscillator with time-independent frequency [33]. The solution of this path integral can be written as

$$
\begin{aligned}
& G^{(\theta)}\left(r_{f}, \varphi_{f}, r_{i}, \varphi_{i}, T\right)=\mathrm{i} \int_{0}^{\infty} \frac{\mathrm{d} e_{0}}{2} \int \frac{\mathrm{d} p_{0}}{2 \pi} \mathrm{e}^{-\mathrm{i} p_{0} T} \\
& \cdot \exp \left\{\mathrm{i} \frac{e_{0}}{2}\left[p_{0}^{2}-m^{2}+2 m \omega\right]\right\} \\
& \cdot \frac{m \omega}{2 \pi \mathrm{i} \sqrt{\omega_{1}} \sin \left(m \omega \sqrt{\omega_{1}} e_{0}\right)} \\
& \cdot \exp \left[-\frac{m \omega}{2 \mathrm{i} \sqrt{\omega_{1}}}\left(r_{i}^{2}+r_{f}^{2}\right) \cot \left(m \omega \sqrt{\omega_{1}} e_{0}\right)\right] \\
& \cdot \sum_{m_{\ell}=0}^{\infty} \exp \left[\mathrm{i} m_{\ell}\left(\varphi_{f}-\varphi_{i}+\frac{(m \omega)^{2} \theta}{2} e_{0}\right)\right] \\
& \cdot I_{m_{\ell}}\left(\frac{m \omega r_{i} r_{f}}{\mathrm{i} \sqrt{\omega_{1}} \sin \left(m \omega \sqrt{\omega_{1}} e_{0}\right)}\right) \cdot
\end{aligned}
$$

For determining the energy-levels and wave functions, we must use the Hille-Hardy formula and the properties of Laguerre polynomials series [33] in (24), then we integrate over the proper time $\frac{\mathrm{d} e_{0}}{2}$. We get finally

$$
\begin{aligned}
& G^{(\theta)}\left(r_{f}, \varphi_{f}, r_{i}, \varphi_{i}, T\right)=-\int \frac{\mathrm{d} p_{0}}{2 \pi} \frac{\mathrm{e}^{-\mathrm{i} p_{0} T}}{p_{0}^{2}-p_{0 n}^{2}} \\
& \cdot \sum_{m_{\ell}} \sum_{n} \Psi_{n, m_{\ell}}^{(\theta)}\left(r_{f}, \varphi_{f}\right) \Psi_{n, m_{\ell}}^{*(\theta)}\left(r_{i}, \varphi_{i}\right),
\end{aligned}
$$

with $\Psi_{n, m_{\ell}}^{(\theta)}(r, \varphi)$ 


$$
\Psi_{n, m_{\ell}}^{(\theta)}(r, \varphi)=\left[\frac{m \omega}{\pi \sqrt{\omega_{1}}} \frac{n !}{\left(n+\left|m_{\ell}\right|\right) !}\right]^{1 / 2}\left(\frac{m \omega}{\sqrt{\omega_{1}}} r^{2}\right)^{\left(m_{\ell}\right) / 2} \exp \left(\operatorname{i} m_{\ell} \varphi-\frac{m \omega}{2 \sqrt{\omega_{1}}} r^{2}\right) L_{n}^{\left(\left|m_{\ell}\right|\right)}\left(\frac{m \omega}{\sqrt{\omega_{1}}} r^{2}\right)
$$

where $L_{n}^{\left(\left|m_{\ell}\right|\right)}$ are generalized Laguerre polynomials. The poles of $G^{(\theta)}\left(r_{f}, \varphi_{f}, r_{i}, \varphi_{i}, T\right)$ yield the discrete energy spectrum

$$
p_{0 n}= \pm \sqrt{2 m \omega \sqrt{\omega_{1}}\left[2 n+\left|m_{\ell}\right|+1\right]-m_{\ell}(m \omega)^{2} \theta+m^{2}-2 m \omega} .
$$

To evaluate the wave functions and energy spectrum, let us integrate over the $p_{0}$ variable. This can be converted to a complex integration along the special contour $C$, and then using the residue theorem, we get

$$
\oint \frac{\mathrm{d} p_{0}}{2 \pi} \frac{\mathrm{e}^{-\mathrm{i} p_{0} T}}{p_{0}^{2}-p_{0 n}^{2}}=-\mathrm{i}\left[\Theta(T) \frac{\mathrm{e}^{-\mathrm{i} E_{n}^{(\theta)} T}}{2 E_{n}^{(\theta)}}+\Theta(-T) \frac{\mathrm{e}^{\mathrm{i} E_{n}^{(\theta)} T}}{2 E_{n}^{(\theta)}}\right],
$$

where the energy eigenvalues are given by

$$
E_{n}^{(\theta)}=\sqrt{2 m \omega \sqrt{1+\frac{(m \omega \theta)^{2}}{4}}\left[2 n+\left|m_{\ell}\right|+1\right]-m_{\ell}(m \omega)^{2} \theta+m^{2}-2 m \omega .}
$$

In (28), we have two types of propagation, one with positive energy $\left(+E_{n}^{(\theta)}\right)$ propagating to the future and the other with negative energy $\left(-E_{n}^{(\theta)}\right)$ propagating to the past. From this result, we deduce the energy spectrum and the corresponding wave functions from (25) by writing

$$
\begin{aligned}
& G^{(\theta)}\left(r_{f}, \varphi_{f}, r_{i}, \varphi_{i} ; T\right)= \\
& -\sum_{n}\left[\Theta(T) \xi_{n}^{(\theta)}\left(r_{f}, \varphi_{f}\right) \xi_{n}^{*(\theta)}\left(r_{i}, \varphi_{i}\right) \mathrm{e}^{-\mathrm{i} E_{n}^{(\theta)} T}\right. \\
& \left.+\Theta(-T) \xi_{n}^{(\theta)}\left(r_{f}, \varphi_{f}\right) \xi_{n}^{*(\theta)}\left(r_{i}, \varphi_{i}\right) \mathrm{e}^{\mathrm{i} E_{n}^{(\theta)} T}\right],
\end{aligned}
$$

where $E_{n}^{(\theta)}$ is defined in (29), and the $\xi_{n}^{(\theta)}(r, \varphi)$ are given by

$$
\begin{aligned}
& \xi_{n}^{(\theta)}(r, \varphi)=\sum_{m_{\ell}} \frac{1}{2 E_{n}^{(\theta)}}\left(\frac{m \omega}{\pi \sqrt{1+\frac{(m \omega \theta)^{2}}{4}}} \frac{n !}{\left(n+\left|m_{\ell}\right|\right) !}\right)^{1 / 2} \\
& \cdot\left(\frac{m \omega}{\sqrt{1+\frac{(m \omega \theta)^{2}}{4}}} r^{2}\right)^{\left(m_{\ell}\right) / 2} \\
& \cdot \exp \left(\mathrm{i} m_{\ell} \varphi-\frac{m \omega}{2}\left(1+\frac{(m \omega \theta)^{2}}{4}\right)^{-1 / 2} r^{2}\right) L_{n}^{\left(\left|m_{\ell}\right|\right)} \\
& \cdot\left(m \omega\left(1+\frac{(m \omega \theta)^{2}}{4}\right)^{-1 / 2} r^{2}\right)
\end{aligned}
$$

with $(r, \varphi)$ commutative space coordinates.

\section{The Green Function of a Dirac Oscillator in a Noncommutative Space Representation}

In order to determine the Dirac oscillator in commutative space, we introduce the nonminimal substitution $p \rightarrow p-\mathrm{i} m \omega x \gamma^{0}$, which was proposed for the first time by Moshinsky and Szczepaniak [34], where $\omega$ is the oscillator frequency, $\gamma^{0}$ the usual Pauli matrices, and $m$ the rest mass of the particle. In this section, we intend to construct the path integral for a twodimensional relativistic Dirac oscillator propagator in a noncommutative space representation following the Fradkin-Gitman method [18, 19]. The propagator of the Dirac oscillator is governed by the causal Green function $S^{c}\left(x_{f}, x_{i}\right)$,

$\left(\gamma^{\mu} \hat{\pi}_{\mu}-m\right) * S^{c}\left(x_{f}, x_{i}\right)=-\delta^{3}\left(x_{f}-x_{i}\right) ;$ $m^{2} \rightarrow m^{2}-\mathrm{i} \varepsilon$.

In a treatment similar to that applied to (13) in Section 2, (32) can be rewritten as

$$
\begin{aligned}
& \left(\gamma^{\mu} \widetilde{\pi}_{\mu}-m\right) S^{(\theta) c}\left(x_{f}, x_{i}\right)=-\delta^{3}\left(x_{f}-x_{i}\right) ; \\
& m^{2} \rightarrow m^{2}-\mathrm{i} \varepsilon .
\end{aligned}
$$

The components $\tilde{\hat{\pi}}$ are

$$
\widetilde{\hat{\pi}}_{0}=\mathrm{i} \frac{\partial}{\partial t}, \quad \widetilde{\hat{\pi}}_{i}=\mathrm{i} \frac{\partial}{\partial x_{i}}-\mathrm{i} m \omega \gamma^{0} \widetilde{\hat{x}}_{i},
$$

where $\tilde{\hat{x}}_{i}=\hat{x}_{i}-\frac{\theta^{i j}}{2} \hat{p}_{j}$ and the coordinates $\left(\hat{x}_{i}, \hat{p}_{i}\right)$ satisfy the usual Heisenberg bracket. The $\gamma$-matrices in 
$(2+1)$ dimensions are usual Dirac matrices which satisfy the standard commutations

$$
\begin{aligned}
& {\left[\gamma^{\mu}, \gamma^{v}\right]_{+}=2 \eta^{\mu v}, \gamma^{\mu} \gamma^{v}=\mathrm{i} \varepsilon^{\mu v \alpha} \gamma^{\alpha}} \\
& \mu, v, \alpha=0,1,2
\end{aligned}
$$

Those Dirac matrices relate to the Pauli matrices, and they obey the relations

$$
\gamma^{0}=\sigma^{3}, \gamma^{1}=\gamma^{0} \sigma^{1} ; \gamma^{2}=\gamma^{0} \sigma^{2}
$$

First, we present $S^{(\theta) c}\left(x_{f}, y_{f}, x_{i}, y_{i}\right)$ as a matrix element of an operator $\hat{S}^{(\theta) c}$,

$$
S^{(\theta) c}\left(x_{f}, y_{f}, x_{i}, y_{i}\right)=\left\langle x_{f} y_{f}\left|\hat{S}^{(\theta) c}\right| x_{i} y_{i}\right\rangle,
$$

where $\hat{S}^{(\theta) c}$ is given by

$$
\begin{aligned}
\hat{S}^{(\theta) c}= & \left(\gamma^{v} \tilde{\hat{\pi}}_{v}+m\right) \\
& \cdot \frac{1}{\left(\gamma^{\mu} \tilde{\hat{\pi}}_{\mu}-m\right)\left(\gamma^{v} \tilde{\hat{\pi}}_{v}+m\right)} .
\end{aligned}
$$

Now, in order to build a global representation propagator, we use the relation $\int\left|x_{f}\right\rangle\left\langle x_{f}\right| \mathrm{d} x_{f}=1$ between $\left(\gamma^{v} \widetilde{\hat{\pi}}_{v}+m\right)$ and $\left[\left(\gamma^{\mu} \widetilde{\hat{\pi}}_{\mu}-m\right)\left(\gamma^{v} \widetilde{\hat{\pi}}_{v}+m\right)\right]^{-1}$. We get

$$
\begin{aligned}
S^{(\theta) c}\left(x_{f}, y_{f}, x_{i}, y_{i}\right)= & \left(\gamma^{v} \tilde{\hat{\pi}}_{v}+m\right)_{\text {out }} \\
& \cdot \tilde{S}^{(\theta) g}\left(x_{f}, y_{f}, x_{i}, y_{i}\right),
\end{aligned}
$$

with

$$
\begin{aligned}
& \tilde{S}^{(\theta) g}\left(x_{f}, y_{f}, x_{i}, y_{i}\right) \\
& \quad=\left\langle x_{f} y_{f}\left|\frac{1}{\left(\gamma^{\mu} \widetilde{\hat{\pi}}_{\mu}-m\right)\left(\gamma^{v} \widetilde{\hat{\pi}}_{v}+m\right)}\right| x_{i} y_{i}\right\rangle .
\end{aligned}
$$

Through the Schwinger proper-time method, $\tilde{S}^{(\theta) g}\left(x_{f}, y_{f}, x_{i}, y_{i}\right)$ is represented as below:

$$
\begin{aligned}
& \tilde{S}^{(\theta) g}\left(x_{f}, y_{f}, x_{i}, y_{i}\right) \\
& =-\mathrm{i} \int_{0}^{\infty} \mathrm{d} \lambda\left\langle x_{f} y_{f}\left|\exp \left[-\mathrm{i} \hat{H}^{(\theta)}(\lambda)\right]\right| x_{i} y_{i}\right\rangle,
\end{aligned}
$$

where the Hamiltonian $\hat{H}^{(\theta)}(\lambda)$ consists of two parts: one is the Hamiltonian of the Dirac oscillator in the commutative space and the second term is considered to be a correction imposed by noncommutativity which depends on $\theta$,

$$
\begin{aligned}
& \hat{H}^{(\theta)}(\lambda)=-\lambda\left(\gamma^{\mu} \widetilde{\hat{\pi}}_{\mu}-m\right)\left(\gamma^{v} \widetilde{\hat{\pi}}_{v}+m\right) \\
& =-\lambda\left[\hat{p}_{0}^{2}-m^{2}-\left(\hat{p}_{x}^{2}+\hat{p}_{y}^{2}\right)-m^{2} \omega^{2}\left(\hat{x}^{2}+\hat{y}^{2}\right)\right. \\
& \left.-\frac{\mathrm{i}}{2} F_{i j}^{(\theta)} \gamma^{i} \gamma^{j}+2 m \omega\left(\hat{x} \hat{p}_{y}-\hat{y} \hat{p}_{x}\right)\right]-\lambda\left(\frac{m \omega \theta}{2}\right) \\
& \cdot\left[2 m \omega\left(\hat{x} \hat{p}_{y}-\hat{y} \hat{p}_{x}\right)-\left(2+\frac{m \omega \theta}{2}\right)\left(\hat{p}_{x}^{2}+\hat{p}_{y}^{2}\right)\right] .
\end{aligned}
$$

So, in order to build a path integral representation for $S^{(\theta) c}\left(x_{f}, y_{f}, x_{i}, y_{i}\right)$, we follow the standard discretization method for the kernel of (41). Usually, we write $\exp \left(-\mathrm{i} \hat{H}^{(\theta)}(\lambda)\right)=\exp \left(-\mathrm{i} \frac{\hat{H}^{(\theta)}(\lambda)}{N+1}\right)^{N+1}$, and we insert $N$ identities $\int|x\rangle\langle x| \mathrm{d} x=1$, between all the operators $\exp \left(-\mathrm{i} \frac{\hat{H}^{(\theta)}(\lambda)}{N+1}\right)$. Next, we introduce $(N+1)$ integrations $\int \mathrm{d} \lambda_{k} \delta\left(\lambda_{k}-\lambda_{k-1}\right)=1$ and $(N+1)$ identities $\int|p\rangle\langle p| \mathrm{d} p=1$. This transforms the expression of $S^{(\theta) c}\left(x_{f}, y_{f}, x_{i}, y_{i}\right)$ into the following Hamiltonian path integral representation:

$S^{(\theta) c}\left(x_{f}, y_{f}, x_{i}, y_{i}, t_{f}, t_{i}\right)=(-\mathrm{i})\left(\gamma^{v} \widetilde{\hat{\pi}}_{v}+m\right)_{\text {out }} \mathbb{T}$

$\cdot \int_{0}^{\infty} \mathrm{d} \lambda_{0} \int D t D x D y \int D p_{0} D p_{x} D p_{y} \int D \lambda D \pi_{\lambda}$

$\cdot \exp \left\{\mathrm{i} \int_{0}^{1}\left[\lambda\left[p_{0}^{2}-m^{2}-\omega_{2}^{2}\left(p_{x}^{2}+p_{y}^{2}\right)\right.\right.\right.$

$-m^{2} \omega^{2}\left(x^{2}+y^{2}\right)-\frac{\mathrm{i}}{2} F_{i j}^{(\theta)} \gamma^{i} \gamma^{j}$

$\left.\left.\left.+2 m \omega \omega_{2}\left(x p_{y}-y p_{x}\right)+p_{x} \dot{x}+p_{y} \dot{y}+p_{0} \dot{t}+\pi_{\lambda} \dot{\lambda}\right]\right] \mathrm{d} s\right\}$,

where $\omega_{2}=1+\frac{m \omega \theta}{2}$ and $F^{(\theta)}$ is an antisymmetric matrix, known as

$F^{(\theta)}=2 m \omega\left(\begin{array}{ccc}0 & 0 & 0 \\ 0 & 0 & -\left(1+\frac{m \omega \theta}{2}\right) \\ 0 & \left(1+\frac{m \omega \theta}{2}\right) & 0\end{array}\right)$

and $\vec{x}(s), \vec{p}(s), \lambda(s)$, and $\pi_{\lambda}(s)$ are bosonic trajectory variables obeying the boundary condition $\vec{x}(0)=\vec{x}_{i}$, $\vec{x}(1)=\vec{x}_{f}, \lambda(0)=\lambda_{0}$. The ordering operator $\mathbb{T}$ acts on the $\gamma$-matrices which are supposed to depend formally on the time parameter $s$. Via a path integral over Grassmannian odd trajectories [18, 19] , the Hamiltonian path integral representation for (44), can be transformed as follows: 


$$
\begin{aligned}
& S^{(\theta) g}\left(x_{f}, y_{f}, x_{i}, y_{i}, T\right)=(-\mathrm{i}) \mathrm{e}^{\mathrm{i} \gamma^{n} \frac{\delta_{l}}{\delta \xi^{n}}} \\
& \cdot \int \frac{\mathrm{d} p_{0}}{2 \pi} \mathrm{e}^{-\mathrm{i} p_{0} T} \int_{0}^{\infty} \mathrm{d} \lambda_{0} \int D x D y \int D p_{x} D p_{y} \int D \lambda D \pi_{\lambda} \\
& \cdot \mathrm{e}^{\mathrm{i} \lambda_{0}\left(p_{0}^{2}-m^{2}\right)} \int_{\psi(0)+\psi(1)=\xi} \mathcal{D} \psi \\
& \cdot \exp \left\{\mathrm { i } \int _ { 0 } ^ { 1 } \left[\lambda \left[-\omega_{2}^{2}\left(p_{x}^{2}+p_{y}^{2}\right)-m^{2} \omega^{2}\left(x^{2}+y^{2}\right)\right.\right.\right. \\
& \left.+2 \mathrm{i} F_{n k}^{(\theta)} \psi^{n} \psi^{k}+2 m \omega \omega_{2}\left(x p_{y}-y p_{x}\right)\right]+p_{x} \dot{x}+p_{y} \dot{y} \\
& \left.\left.+\pi_{\lambda} \dot{\lambda}-\mathrm{i} \psi_{n} \dot{\psi}^{n}\right] \mathrm{~d} s+\psi_{n}(1) \psi^{n}(0)\right\}_{\xi=0},
\end{aligned}
$$

where the measure $\mathcal{D} \psi$ is given by

$\mathcal{D} \psi=D \psi$

$$
\cdot\left[\int_{\psi(0)+\psi(1)=0} D \psi \exp \left(\int_{0}^{1} \psi_{n} \dot{\psi}^{n} \mathrm{~d} s\right)\right]^{-1}
$$

and $\xi_{n}$ are auxiliary Grassmann (odd) variables, anticommuting by definition with the $\gamma$-matrices; $\psi_{n}(s)$ are odd trajectories of integration. First, one can integrate over $\pi_{\lambda}(s)$, and then using the arising $\delta$-functions to remove the functional integration over $\lambda$. It is important to replace the integration over $\psi$ by the odd velocities $\omega$, because of the boundary conditions $\psi^{n}(0)+$ $\psi^{n}(1)=\xi^{n}$. Following the replacement,

$\psi(s)=\frac{1}{2} \int_{0}^{1} \varepsilon\left(s-s^{\prime}\right) \omega\left(s^{\prime}\right) \mathrm{d} s^{\prime}+\frac{\xi}{2}$,

with $\varepsilon(s)$ the sign of $s$. Then (46) becomes

$S^{(\theta) g}\left(x_{f}, y_{f}, x_{i}, y_{i}, T\right)=(-\mathrm{i}) \exp \left(\mathrm{i} \gamma^{n} \frac{\delta_{l}}{\delta \xi^{n}}\right)$

$\int \frac{\mathrm{d} p_{0}}{2 \pi} \mathrm{e}^{-\mathrm{i} p_{0} T} \int_{0}^{\infty} \mathrm{d} \lambda_{0} \exp \left(\mathrm{i} \lambda_{0}\left(p_{0}^{2}-m^{2}\right)\right)$

$\cdot \int D x D y \int D p_{x} D p_{y} \int \mathcal{D} \omega$

$$
\begin{aligned}
& \cdot \exp \left\{\mathrm { i } \int _ { 0 } ^ { 1 } \left[\lambda _ { 0 } \left[-\omega_{2}^{2}\left(p_{x}^{2}+p_{y}^{2}\right)-(m \omega)^{2}\left(x^{2}+y^{2}\right)\right.\right.\right. \\
& \left.+2 m \omega \omega_{2}\left(x p_{y}-y p_{x}\right)\right]-\frac{\mathrm{i} \lambda_{0}}{2}\left(\omega^{n} \varepsilon-\xi^{n}\right) \\
& \left.\left.\cdot F_{n k}^{(\theta)}\left(\varepsilon \omega^{k}+\xi^{k}\right)+\frac{\mathrm{i}}{2} \omega_{n} \varepsilon \omega^{n}+p_{x} \dot{x}+p_{y} \dot{y}\right] \mathrm{~d} s\right\}_{\xi=0},
\end{aligned}
$$

where the measure $\mathcal{D} \omega$ is

$$
\mathcal{D} \omega=D \omega\left[\int D \omega \exp \left(-\frac{1}{2} \omega^{n} \varepsilon \omega_{n}\right)\right]^{-1} \text {. }
$$

Through (49), we can see that the path integral in Grassmann variables is Gaussian, which is clearly shown in the appendix. Following the same steps of the account in the previous Section 2 (as in [33]), we can find $S^{(\theta) g}$ :

$S^{(\theta) g}\left(r_{f}, \varphi_{f}, r_{i}, \varphi_{i}, T\right)=$

$(-\mathrm{i}) \sum_{n} \sum_{m_{\ell}} \int_{0}^{\infty} \frac{\mathrm{d} e_{0}}{2} \int \frac{\mathrm{d} p_{0}}{2 \pi} \mathrm{e}^{\mathrm{i} \frac{e_{0}}{2}\left(p_{0}^{2}-m^{2}\right)-\mathrm{i} p_{0} T}$

$\cdot \mathrm{e}^{-\mathrm{i}\left[e_{0} m \omega\left(1+\frac{m \omega \theta}{2}\right)\left(\left(2 n+\left|m_{\ell}\right|+1\right)-m_{\ell}\right)\right]}$

.$\Psi_{n, m_{\ell}}^{(\theta)}\left(r_{f}, \varphi_{f}\right) \Psi_{n, m_{\ell}}^{(\theta) *}\left(r_{i}, \varphi_{i}\right)$

- $\left[\cos \left(2 m \omega \omega_{2} e_{0}\right)+\gamma^{0} \sin \left(2 m \omega \omega_{2} e_{0}\right)\right]$,

where $\Psi_{n, m_{\ell}}^{(\theta)}(r, \varphi)$ is given by

$\Psi_{n, m_{\ell}}^{(\theta)}(r, \varphi)=\left[\frac{m \omega}{\pi \omega_{2}} \frac{n !}{\left(n+\left|m_{\ell}\right|\right) !}\right]^{1 / 2}\left(\frac{m \omega}{\omega_{2}} r^{2}\right)^{\left(m_{\ell}\right) / 2}$

$\cdot \exp \left(\mathrm{i} m_{\ell} \varphi-\frac{m \omega}{2 \omega_{2}} r^{2}\right) L_{n}^{\left(\left|m_{\ell}\right|\right)}\left(\frac{m \omega}{\omega_{2}} r^{2}\right)$.

$L_{n}^{\left(\left|m_{\ell}\right|\right)}$ is a generalized Laguerre polynomial, and $m_{\ell}$ is an integer number. In addition to that, we integrate over $\frac{\mathrm{d} e_{0}}{2}$, then we act through the operator $\left(\gamma^{v} \widetilde{\hat{\pi}}_{v}+m\right)_{\text {out }}$. Finally, we use the properties of Laguerre's polynomials [35] to obtain:

$$
\begin{aligned}
& S^{(\theta) c}\left(r_{f}, \varphi_{f}, r_{i}, \varphi_{i}\right)=\sum_{n \in N} \sum_{m_{\ell} \in \mathcal{Z}} \frac{1}{z_{f}^{1 / 4} z_{i}^{1 / 4}} \mathrm{e}^{-\frac{\left(z_{f}+z_{i}\right)}{2}}\left[\frac{m \omega}{\omega_{2}} \frac{n !}{\left(n+\left|m_{\ell}\right|\right) !}\right] \int \frac{\mathrm{d} p_{0}}{2 \pi} \frac{\mathrm{e}^{-i p_{0} T}}{p_{0}^{2}-p_{n}^{2}} \\
& {\left[\begin{array}{cc}
\left(p_{0}+m\right)\left(z_{f}\right)^{\left(m_{\ell}+\frac{1}{2}\right) / 2}\left(z_{i}\right)^{\left(m_{\ell}+\frac{1}{2}\right) / 2} & \left(2 \mathrm{i} \sqrt{\frac{m \omega}{n \omega_{2}}}\right)\left(z_{f}\right)^{\left(m_{\ell}+\frac{1}{2}\right) / 2} \mathrm{e}^{\mathrm{i} m_{\ell} \varphi_{f}} L_{n}^{\left|m_{\ell}\right|}\left(z_{f}\right) \\
\cdot \mathrm{e}^{\mathrm{i} m_{\ell}\left(\varphi_{f}-\varphi_{i}\right)} L_{n}^{\left(\left|m_{\ell}\right|\right)}\left(z_{i}\right) L_{n}^{\left(\left|m_{\ell}\right|\right)}\left(z_{f}\right) & \cdot\left(z_{i}\right)^{\left(m_{\ell}+\frac{3}{2}\right) / 2} \mathrm{e}^{\mathrm{i}\left(m_{\ell}+1\right) \varphi_{i}} L_{n-1}^{\left|m_{\ell}+1\right|}\left(z_{i}\right) \\
\left(2 \mathrm{i} \sqrt{\frac{m \omega}{n \omega_{2}}}\right) \mathrm{e}^{\mathrm{i}\left(m_{\ell}+1\right) \varphi_{f}\left(z_{f}\right)^{\left(m_{\ell}+\frac{3}{2}\right) / 2} L_{n-1}^{\left|m_{\ell}+1\right|}\left(z_{f}\right)} & \frac{1}{n}\left(-p_{0}+m\right)\left(z_{f}\right)^{\left(m_{\ell}+\frac{3}{2}\right) / 2}\left(z_{i}\right)^{\left(m_{\ell}+\frac{3}{2}\right) / 2} \\
\cdot \mathrm{e}^{-\mathrm{i} m_{\ell} \varphi_{i}}\left(z_{i}\right)^{\left(m_{\ell}+\frac{1}{2}\right) / 2} L_{n}^{\left|m_{\ell}\right|}\left(z_{i}\right) & \cdot \mathrm{e}^{\mathrm{i}\left(m_{\ell}+1\right)\left(\varphi_{f}-\varphi_{i}\right)} L_{n-1}^{\left(\left|m_{\ell}+1\right|\right)}\left(z_{i}\right) L_{n-1}^{\left(\left|m_{\ell}+1\right|\right)}\left(z_{f}\right)
\end{array}\right],}
\end{aligned}
$$


where $z=\frac{m \omega}{\omega_{2}} r^{2}$ and

$p_{n}^{2}=m^{2}+2 m \omega \omega_{2}\left(2 n+\left|m_{\ell}\right|+1\right)$

$$
-m^{2} \omega^{2} \theta\left(m_{\ell}+1\right)-2 m \omega m_{\ell}-2 m \omega .
$$

The determination of the wave functions is performed by applying the residue theorem. Let's choose a special contour $C$ in the complex plane. The poles of the Green function are positive energies and negative energies are given respectively by $p_{+}^{0}=E_{n}-\mathrm{i} \varepsilon, p_{-}^{0}=-E_{n}+\mathrm{i} \varepsilon$. For positive energies $p_{+}^{0}$, the contour of integration is
H. Benzair et al. · Relativistic Oscillators: a Path Integral Approach

chosen below the real axis with $T>0$. On the other hand, for negative energies $p_{-}^{0}$, it is chosen above the real axis with $T<0$. In conclusion, we have

$$
\begin{aligned}
& \oint \frac{\mathrm{d} p_{0}}{2 \pi} \frac{\mathrm{e}^{-\mathrm{i} p_{0} T}}{p_{0}^{2}-p_{0 n}^{2}}= \\
& -\mathrm{i}\left[\Theta(T) \frac{\mathrm{e}^{-\mathrm{i} E_{n}^{(\theta)} T}}{2 E_{n}^{(\theta)}}+\Theta(-T) \frac{\mathrm{e}^{\mathrm{i} E_{n}^{(\theta)} T}}{2 E_{n}^{(\theta)}}\right]
\end{aligned}
$$

and

$$
\oint \frac{\mathrm{d} p_{0}}{2 \pi} \frac{\mathrm{e}^{-\mathrm{i} p_{0} T}}{p_{0}^{2}-p_{0 n}^{2}}\left(p_{0} \pm m\right)=-\mathrm{i}\left[\Theta(T) \frac{\left(E_{n}^{(\theta)} \pm m\right)}{2 E_{n}^{(\theta)}} \mathrm{e}^{-\mathrm{i} E_{n}^{(\theta)} T}+\Theta(-T) \frac{\left(E_{n}^{(\theta)} \mp m\right)}{2 E_{n}^{(\theta)}} \mathrm{e}^{\mathrm{i} E_{n}^{(\theta)} T}\right],
$$

where

$$
E_{n}^{(\theta)}=\sqrt{m^{2}+2 m \omega \omega_{2}\left(2 n+\left|m_{\ell}\right|+1\right)-m^{2} \omega^{2} \theta\left(m_{\ell}+1\right)-2 m \omega m_{\ell}-2 m \omega} .
$$

In (55), we have two types of propagation, one with positive energy $+E_{n}^{(\theta)}$ propagating to the future and the other with negative energy $-E_{n}^{(\theta)}$ propagating to the past. We get the propagator in NC space but defined with commutative variables:

$$
\begin{aligned}
& S^{(\theta)}\left(r_{f}, \varphi_{f}, r_{i}, \varphi_{i} ; T\right)= \\
& \sum_{n} \sum_{m_{\ell}}\left[\Theta(T) \Psi_{n, m_{\ell}}^{(\theta)+}\left(r_{f}, \varphi_{f}\right) \bar{\Psi}_{n, m_{\ell}}^{(\theta)+}\left(r_{i}, \varphi_{i}\right) \mathrm{e}^{-\mathrm{i} E_{n}^{(\theta)} T}+\Theta(-T) \Psi_{n, m_{\ell}}^{(\theta)-}\left(r_{f}, \varphi_{f}\right) \bar{\Psi}_{n, m_{\ell}}^{(\theta)-}\left(r_{i}, \varphi_{i}\right) \mathrm{e}^{\mathrm{i} E_{n}^{(\theta)} T}\right] .
\end{aligned}
$$

From this result, we deduce the energy spectrum with $\omega_{2}=1+\frac{m \omega \theta}{2}$ in (57),

$$
E_{n}^{(\theta)}= \pm \sqrt{m^{2}+2 m \omega\left(1+\frac{m \omega \theta}{2}\right)\left(2 n+\left|m_{\ell}\right|+1\right)-m^{2} \omega^{2} \theta\left(m_{\ell}+1\right)-2 m \omega m_{\ell}-2 m \omega}
$$

or otherwise

$$
E_{n}^{(\theta)}= \pm \sqrt{m^{2}+2 n\left(2 m \omega+m^{2} \omega^{2} \theta\right)+\left|m_{\ell}\right|\left(2 m \omega+m^{2} \omega^{2} \theta\right)(1 \mp 1)} .
$$

The corresponding wave functions are

$$
\begin{aligned}
& \Psi_{n, m_{\ell}}^{(\theta)+}(r, \varphi)=\frac{1}{\left(\frac{m \omega}{\omega_{2}} r^{2}\right)^{1 / 4}}\left(\begin{array}{c}
\mathrm{e}^{\mathrm{i} m_{\ell} \varphi} \psi_{1}^{(\theta)+}(r) \\
\mathrm{e}^{\mathrm{i}\left(m_{\ell}+1\right) \varphi} \psi_{2}^{(\theta)+}(r)
\end{array}\right), \\
& \Psi_{n, m_{\ell}}^{(\theta)-}(r, \varphi)=\frac{1}{\left(\frac{m \omega}{\omega_{2}} r^{2}\right)^{1 / 4}}\left(\begin{array}{c}
\mathrm{e}^{\mathrm{i} m_{\ell} \varphi} \psi_{1}^{(\theta)-}(r) \\
\mathrm{e}^{\mathrm{i}\left(m_{\ell}+1\right) \varphi} \psi_{2}^{(\theta)-}(r)
\end{array}\right) .
\end{aligned}
$$

The components of the wave functions $\Psi_{n, m_{\ell}}^{(\theta)+}(r, \varphi)$ and $\Psi_{n, m_{\ell}}^{(\theta)-}$ are respectively

$$
\begin{aligned}
& \psi_{1, n, m_{\ell}}^{(\theta)+}(r)=\left[\sqrt{\frac{m \omega}{\pi \omega_{2}}} \frac{n !}{\pi\left(n+\left|m_{\ell}\right|\right) !} \frac{E_{n}^{(\theta)}+m}{2 E_{n}^{(\theta)}}\right]^{1 / 2} \\
& \cdot\left(\frac{m \omega}{\omega_{2}} r^{2}\right)^{\left(m_{\ell}+1 / 2\right) / 2} \mathrm{e}^{-\frac{m \omega r^{2}}{2 \omega_{2}}} L_{n}^{\left(\left|m_{\ell}\right|\right)}\left(\frac{m \omega}{\omega_{2}} r^{2}\right), \\
& \psi_{2, n, m_{\ell}}^{(\theta)+}(r)=\mathrm{i}\left[\sqrt{\frac{m \omega}{\pi \omega_{2}}} \frac{(n-1) !}{\pi\left(n+\left|m_{\ell}\right|\right) !} \frac{E_{n}^{(\cdot \theta)}-m}{E_{n}^{(\theta)}}\right]^{1 / 2}
\end{aligned}
$$$$
\cdot\left(\frac{m \omega}{\omega_{2}} r^{2}\right)^{\left(m_{\ell}+3 / 2\right) / 2} \mathrm{e}^{-\frac{m \omega r^{2}}{2 \omega_{2}}} L_{n-1}^{\left(\left|m_{\ell}+1\right|\right)}\left(\frac{m \omega}{\omega_{2}} r^{2}\right),
$$ 


$$
\begin{aligned}
& \psi_{1, n, m_{\ell}}^{(\theta)-}(r)=\left[\sqrt{\frac{m \omega}{\pi \omega_{2}}} \frac{n !}{\pi\left(n+\left|m_{\ell}\right|\right) !} \frac{E_{n}^{(\theta)}-m}{2 E_{n}^{(\theta)}}\right]^{1 / 2} \\
& \cdot\left(\frac{m \omega}{\omega_{2}} r^{2}\right)^{\left(m_{\ell}+1 / 2\right) / 2} \mathrm{e}^{-\frac{m \omega r^{2}}{2 \omega_{2}}} L_{n}^{\left(\left|m_{\ell}\right|\right)}\left(\frac{m \omega}{\omega_{2}} r^{2}\right) \\
& =\sqrt{\frac{E_{n}^{(\theta)}-m}{E_{n}^{(\theta)}+m}} \psi_{1, n, m_{\ell}}^{(\theta)+}(r), \\
& \psi_{2, n, m_{\ell}}^{(\theta)-}(r)=\mathrm{i}\left[\sqrt{\frac{m \omega}{\pi \omega_{2}}} \frac{(n-1) !}{\pi\left(n+\left|m_{\ell}\right|\right) !} \frac{E_{n}^{(\theta)}+m}{E_{n}^{(\theta)}}\right]^{1 / 2} \\
& \cdot\left(\frac{m \omega}{\omega_{2}} r^{2}\right)^{\left(m_{\ell}+3 / 2\right) / 2} \mathrm{e}^{-\frac{m \omega r^{2}}{2 \omega_{2}}} L_{n-1}^{\left(\left|m_{\ell}+1\right|\right)}\left(\frac{m \omega}{\omega_{2}} r^{2}\right) \\
& =-\sqrt{\frac{E_{n}^{(\theta)}+m}{E_{n}^{(\theta)}-m}} \psi_{2, n, m_{\ell}}^{(\theta)+}(r) .
\end{aligned}
$$

Now let us discuss two key points which we jumped before. The transformation (16) is not unitary and takes us from a reducible representation in $\widehat{\tilde{x}}_{i}$ to an irreducible one in $\hat{x}_{i}$, so we can represent the operators $\widehat{\tilde{x}}_{i}$ on the Hilbert space of an irreducible one by the following action:

$\widehat{\tilde{x}}_{i} \psi(\mathbf{x})=\left(x_{i}+\mathrm{i} \frac{\theta_{i j}}{2} \partial_{j}\right) \psi(\mathbf{x})$, with $i, j=1,2$,

which indicates that the noncommutative position is nonlocal in this representation. This main difficulty can be turned away by working whether in the momentum basis $p_{i}$ or in the mixed basis of phase space $\left(\tilde{x}, p_{y}\right)$, or alternatively by $\left(\tilde{y}, p_{x}\right)$ [24], whether in the holomorphic representation of raising-lowering operators in terms of this noncommutative coordinates [36, 37]. In [23], it is suggested that we can also move to integration over trajectories as $\tilde{x}=x-\frac{\theta p}{2}$ in the path integrals (19) and (41); then we have

$$
\begin{aligned}
& G^{(\theta)}=-\mathrm{i} \int_{0}^{\infty} \mathrm{d} \lambda \int D p \int_{\vec{x}_{i}-\frac{\theta p}{2}}^{\vec{x}_{f}-\frac{\theta p}{2}} D \tilde{x} \\
& \cdot \exp \left\{\mathrm{i} \int_{0}^{\lambda} \mathrm{d} s\left[p_{i} \tilde{\dot{x}}^{i}-\hat{H}(\tilde{x}, p)+\frac{\dot{p}_{i} \theta^{i j} p_{j}}{2}\right]\right\}
\end{aligned}
$$

and

$$
\begin{aligned}
& S^{(\theta) c}=-\mathrm{i}\left(\gamma^{v} \widetilde{\hat{\pi}}_{v}+m\right)_{\text {out }} \int_{0}^{\infty} \mathrm{d} \lambda \int D p \int_{\vec{x}_{i}-\frac{\theta p}{2}}^{\vec{x}_{f}-\frac{\theta p}{2}} D \tilde{x} \\
& \cdot \exp \left\{\mathrm{i} \int_{0}^{\lambda} \mathrm{d} s\left[p_{i} \widetilde{\dot{x}}^{i}-\hat{H}(\tilde{x}, p, \gamma)+\frac{\dot{p}_{i} \theta^{i j} p_{j}}{2}\right]\right\} .
\end{aligned}
$$

These actions in (67) and (68) are no local and differ from the corresponding commutative case (at $\theta=0$ ) by the term $\frac{1}{2} \dot{p}_{i} \theta^{i j} p_{j}$. This suggestion is not correct in itself and can lead us to some ambiguities because it does not respect the Feynman spirit of the meaning of path integrals. In our opinion, the formulation given in [37] is better. Finally, the commutative representation give us the correct spectrum and some physics properties of the system in the easier and direct manner.

Moreover, if we consider the problem of a charged particle on a noncommutative plane subjected to a constant magnetic field $\mathcal{B}$, where the associated magnetic vector potential $\vec{A}$ is written in a symmetric gauge,

$$
\vec{A}=\frac{\mathcal{B}}{2}(-\tilde{y} \vec{i}+\tilde{x} \vec{j}) .
$$

One can obtain in this case, the Green function representation, the energy and wave functions for the $(2+1)$-dimension Dirac and Klein-Gordon oscillators in a noncommutative space representation by following the same way as in previous calculations and just replacing the frequency $\omega$ by $\varpi \rightarrow \omega+\frac{e \mathcal{B}}{2 m}$.

It leads to $\theta=0$ : the NC space returns to the commutative space, and we recover the same results as for the commutative space case $[38,39]$.

\section{Conclusion}

In this paper, we have shown explicit calculations of Dirac and Klein-Gordon oscillators in noncommutative space by means of supersymmetric path integrals. Exact solutions are obtained and compared with the Dirac and Klein-Gordon oscillator particles subjected to the interaction of a constant magnetic field. The explicit calculation of the Green function in fermionic case became possible and the wave functions were easily determined by following a detailed demonstration of the Fradkin-Gitman method. The propagator calculation has been performed through a representation named global projection. For this case a new term in the action will appear which can be interpreted as a self-interaction for a charged particle with dipole electric and magnetic moments. We could study those systems in the presence of a constant magnetic field $\mathcal{B}$. The exact expression of the energy spectrum and corresponding eigenfunctions expressed in terms of generalized Laguerre polynomials are then deduced with the presence and the absence of the field. Our results coin- 
cide with those obtained in [29]. In the limit $\theta \rightarrow 0$, we recover the same results as in $[38,39]$ (in commutative space).

\section{Appendix A: The Integration over the Spin Variables}

For (49), the Polykov spin factor will be given by

$$
\begin{aligned}
& \mathcal{I}_{\text {spin }}^{(\theta)}=\mathrm{e}^{\left(-\frac{\lambda_{0}}{2} F^{(\theta)}(\theta)_{n k} \xi^{n} \xi^{k}\right)} \\
& \cdot \int \mathcal{D} \omega \exp \left[-\frac{1}{2} \omega^{n} T_{n k}^{(\theta)} \omega^{k}+I_{n}^{(\theta)} \omega^{n}\right]_{\xi=0}, \\
& I_{k}^{(\theta)}=-\lambda_{0} \xi^{n} F_{n k}^{(\theta)} \varepsilon \\
& T_{n k}^{(\theta)}=\eta_{n k} \varepsilon-\lambda_{0} \varepsilon F_{n k}^{(\theta)} \varepsilon .
\end{aligned}
$$

The integration over $\omega$ has a Gaussian form and gives

$$
\begin{aligned}
& \mathcal{I}_{\text {spin }}^{(\theta)}=\mathrm{e}^{\left(-\frac{\lambda_{0}}{2} F_{n k}^{(\theta)} \xi^{n} \xi^{k}\right)} \sqrt{\frac{\operatorname{det} T^{(\theta)}}{\operatorname{det} \varepsilon}} \\
& \cdot \exp \left[-\frac{1}{2} I_{n}^{(\theta)}\left[\left(T^{(\theta)}\right)^{-1}\right]^{n k} I_{k}^{(\theta)}\right]_{\xi=0} .
\end{aligned}
$$

And $\left(T^{(\theta)}\right)^{-1}$ is the inverse of $T^{(\theta)}$, taking into account its original definition[18-26],

$$
\begin{aligned}
& I_{n}^{(\theta)}\left[\left(T^{(\theta)}\right)^{-1}\right]^{n k} I_{k}^{(\theta)} \\
& \quad=2 \lambda_{0}^{2}\left(F^{(\theta)} \mathcal{G}^{(\theta)} F^{(\theta)}\right)_{m l} \xi^{m} \xi^{l} .
\end{aligned}
$$

One can demonstrate that

$$
\begin{aligned}
& {\left[\frac{\operatorname{det} T^{(\theta)}\left(\lambda_{0}\right)}{\operatorname{det} \varepsilon}\right]} \\
& =\exp \left[-\int_{0}^{\lambda_{0}} \mathrm{~d} \lambda_{0}^{\prime} \operatorname{Tr} \mathcal{G}^{(\theta)}\left(\lambda_{0}^{\prime}\right) F^{(\theta)}(x)\right] .
\end{aligned}
$$

So, we are going to calculate the inverse matrix $T_{n k}^{(\theta)}$, we have

$$
\begin{aligned}
& T_{n k}^{(\theta)}\left(s, s^{\prime}\right)=\varepsilon\left(s-s^{\prime}\right) \eta_{n k} \\
& -\lambda_{0} \int_{0}^{1} \varepsilon(s-\tau) F_{n k}^{(\theta)} \varepsilon\left(\tau-s^{\prime}\right) \mathrm{d} \tau .
\end{aligned}
$$

To get the function

$$
\mathcal{G}_{\alpha \beta}^{(\theta)}\left(s, s^{\prime}\right)=\frac{1}{2} \int_{0}^{1} \Omega_{\alpha \beta}^{(\theta)}\left(s, s^{\prime}\right) \varepsilon\left(\tau-s^{\prime}\right) \mathrm{d} \tau,(\mathrm{A} 6)
$$

it is convenient to first define the function $\Omega_{\alpha \beta}^{(\theta)}\left(s, s^{\prime}\right)$ by

$\Omega_{\alpha \beta}^{(\theta)}\left(s, s^{\prime}\right)=\int_{0}^{1} \varepsilon(s-\tau)\left(T^{(\theta)}\right)_{\alpha \beta}^{-1}\left(\tau, s^{\prime}\right) \mathrm{d} \tau$.

Now, we have

$\int_{0}^{1} T_{n k}^{(\theta)}(s, \tau)\left(T^{(\theta)}\right)_{k \beta}^{-1}\left(\tau, s^{\prime}\right) \mathrm{d} \tau=\delta_{n}^{\beta} \delta\left(s-s^{\prime}\right)$.

And substituting (A5) into (A8), we can easily obtain

$$
\begin{aligned}
& \Omega_{n \beta}^{(\theta)}\left(s, s^{\prime}\right)-\lambda_{0} \int_{0}^{1} \varepsilon(s-\tau) F_{n k}^{(\theta)} \Omega_{\beta}^{(\theta)} k\left(\tau, s^{\prime}\right) \mathrm{d} \tau \\
& =\eta_{n \beta} \delta\left(s-s^{\prime}\right) .
\end{aligned}
$$

This equation is equivalent to the differential equation

$$
\begin{aligned}
& \frac{\mathrm{d} \Omega_{n \beta}^{(\theta)}\left(s, s^{\prime}\right)}{\mathrm{d} s}-2 \lambda_{0} F_{n k}^{(\theta)} \Omega_{\beta}^{(\theta)} k\left(s, s^{\prime}\right) \\
& =\eta_{n \beta} \frac{\mathrm{d} \delta\left(s-s^{\prime}\right)}{\mathrm{d} s}
\end{aligned}
$$

with the initial condition

$$
\begin{aligned}
& \Omega_{n \beta}^{(\theta)}\left(0, s^{\prime}\right)+\lambda_{0} \int_{0}^{1} F_{n k}^{(\theta)} \Omega_{\beta}^{(\theta)} k\left(\tau, s^{\prime}\right) \mathrm{d} \tau \\
& =\eta_{n \beta} \delta\left(s^{\prime}\right) .
\end{aligned}
$$

For (A10) and (A11), we find the solution

$$
\begin{aligned}
\Omega^{(\theta)}\left(s, s^{\prime}\right)= & \delta\left(s-s^{\prime}\right)+\lambda_{0} F^{(\theta)} \mathrm{e}^{2 \lambda_{0} F^{(\theta)}\left(s-s^{\prime}\right)} \\
& \cdot\left[\varepsilon\left(s-s^{\prime}\right)-\tanh \lambda_{0} F^{(\theta)}\right] .
\end{aligned}
$$

Inserting (A12) into (A6), we obtain

$$
\begin{aligned}
\mathcal{G}^{(\theta)}\left(s, s^{\prime}\right)= & \frac{1}{2} \mathrm{e}^{2 \lambda_{0} F^{(\theta)}\left(s-s^{\prime}\right)} \\
& \cdot\left[\varepsilon\left(s-s^{\prime}\right)-\tanh \lambda_{0} F^{(\theta)}\right] .
\end{aligned}
$$

According to (A7), we get

$$
\begin{aligned}
& \left(T^{(\theta)}\right)^{-1}\left(\tau-\tau^{\prime}\right)=\varepsilon^{-1}\left(\tau, \tau^{\prime}\right) \\
& +\left(\lambda_{0} F^{(\theta)}\right)^{2} \mathrm{e}^{2 \lambda_{0} F^{(\theta)}}\left(\tau-\tau^{\prime}\right) \\
& +\left[\varepsilon\left(\tau-\tau^{\prime}\right)-\tanh \left(\lambda_{0} F^{(\theta)}\right)\right] \\
& +\lambda_{0} F^{(\theta)} \mathrm{e}^{2 \lambda_{0} F^{(\theta)}\left(\tau-\tau^{\prime}\right)} \delta\left(\tau-\tau^{\prime}\right) .
\end{aligned}
$$


Substituting (A3) and (A4) into (A2), we obtain

$$
\begin{aligned}
& \mathcal{I}_{\text {spin }}^{(\theta)}=\exp \left(\mathrm{i} \gamma^{n} \frac{\delta_{l}}{\delta \xi^{n}}\right) \\
& \cdot \exp \left\{\mathrm { i } \int _ { 0 } ^ { 1 } \left[2 \mathrm{i} \int_{0}^{\lambda_{0}} \mathrm{~d} \lambda_{0}^{\prime} \operatorname{Tr} \mathcal{G}^{(\theta)}\left(\lambda_{0}^{\prime}\right) F^{(\theta)}\right.\right. \\
& \left.\left.+\mathrm{i} \frac{\lambda_{0}}{2}\left(F^{(\theta)} \mathcal{K}^{(\theta)}\right)_{n k} \xi^{n} \xi^{k}\right] \mathrm{~d} s\right\} \mid \xi=0
\end{aligned}
$$

where

$$
\begin{aligned}
& \mathcal{K}_{n k}^{(\theta)}=\eta_{n k}+2 \lambda_{0}\left(\mathcal{G}^{(\theta)} F^{(\theta)}\right)_{n k}, \\
& \left(F^{(\theta)} \mathcal{K}^{(\theta)}\right)_{n k}=\frac{2}{\lambda_{0}} \tanh \left(\lambda_{0} F^{(\theta)}\right) .
\end{aligned}
$$

And after a straightforward calculation, we get

$$
\begin{aligned}
\mathcal{I}_{\text {spin }}^{(\theta)}= & \exp \left(\mathrm{i} \gamma^{n} \frac{\delta_{l}}{\delta \xi^{n}}\right) \cos \left(\lambda_{0} F^{(\theta)}\right) \\
& \cdot\left[1+\tanh \left(\lambda_{0} F^{(\theta)}\right)_{n k} \xi^{n} \xi^{k}\right]_{\xi=0} .
\end{aligned}
$$

[1] M. Dubois Violette, R. Kerner, and J. Madore, J. Math. Phys. 31, 323 (1990).

[2] A. Connes, Noncommutative Geometry, New York, London 1994.

[3] M. R. Douglas and N. A. Nekrasov, Rev. Mod. Phys. 73, 977 (2001).

[4] H. Weyl, Z. Phys. 46, 1 (1927).

[5] A. H. Chamseddine and A. Connes, Commun. Math. Phys. 186, 731 (1997).

[6] H. Figueroa, J. M. Gracia-Bondia, F. Lizzi, and J. C. Varilly, J. Geom. Phys. 26, 329 (1998).

[7] E. Witten, Nucl. Phys. B 460, 33 (1996).

[8] A. Connes, M. R. Douglas, and A. Schwarz, J. High Energy Phys. 02, 003 (1998).

[9] N. Seiberg and E. Witten, J. High Energy Phys. 09, 032 (1999).

[10] K. Li and J. Wang, Eur. Phys. J. C 50, 1007 (2007).

[11] M. Rosenbaum, J. David Vergara, and L. Roman Juarez, Phys. Lett. A 367, 1 (2007).

[12] J. Jing, S.-H. Zhao, J.-F. Chen, and Z.-W. Long, Eur. Phys. J. C 54, 685 (2008).

[13] M. Falek and M. Merad, Commun. Theor. Phys. 50, 587 (2008)

[14] A. Kijanka and P. Kosiński, Phys. Rev. D 70, 127702 (2004).

[15] M. Chaichian, M. M. Sheikh-Jabbari, and A. Tureanu, Phys. Rev. Lett. 86, 2716 (2001). 26, 2001.
In addition to that, we use the Grassmann algebra by the following identity:

$$
\begin{aligned}
& \left.\exp \left[\mathrm{i} \gamma^{n} \frac{\partial_{l}}{\partial \xi^{n}}\right] f(\xi)\right|_{\xi=0} \\
& =\left.f\left(\frac{\delta}{\delta \zeta^{n}}\right) \exp \left(\mathrm{i} \zeta_{n} \gamma^{n}\right)\right|_{\xi=0} \\
& =\sum_{k=0 n_{1} \ldots n_{k}}^{2} f_{n_{1} \ldots n_{2}} \\
& \left.\cdot \frac{\partial}{\partial \zeta_{n_{1}}} \ldots \frac{\partial}{\partial \zeta_{n_{k}}} \sum_{l=0}^{2} \frac{i^{l}}{l !}\left(\zeta_{n} \gamma^{n}\right)^{l}\right|_{\zeta=0} .
\end{aligned}
$$

Therefore,

$$
\begin{aligned}
\mathcal{I}_{\text {spin }}^{(\theta)}= & {\left[\cos \left(m \omega\left(1+\frac{m \omega \theta}{2}\right) e_{0}\right)\right.} \\
& \left.+\gamma^{0} \sin \left(m \omega\left(1+\frac{m \omega \theta}{2}\right) e_{0}\right)\right]
\end{aligned}
$$

with $\lambda_{0}=e_{0} / 2$.

[16] J. Gamboa, M. Loewe, and J. C. Rojas, Phys. Rev. D. 64, 067901 (2001).

[17] S. Bellucci, A. Nersessian, and C. Sochichiu, Phys. Lett. B 522, 345 (2001).

[18] E. S. Fradkin, Nucl. Phys. 76, 588 (1966).

[19] F. A. Berezin and M. S. Marinov, JETP Lett. 21, 320 (1975).

[20] F. A. Berezin and M. S. Marinov, Ann. Phys. 104, 336 (1977).

[21] E. S. Fradkin and D. M. Gitman, Phys. Rev. D 44, 3230 (1991).

[22] C. Alexandrou, R. Rosenfelder, and A. W. Schreiber, Phys. Rev. A 59, 1762 (1999).

[23] D. M. Gitman and V. G. Kupriyanov, Eur. Phys. J. C 54, 325 (2008).

[24] C. Acatrinei, J. High Energy Phys. 0109, 007 (2001).

[25] D. M. Gitman, S. I. Zlatev, and W. D. Cruz, J. Phys. 26, 419 (1996).

[26] D. M. Gitman and S. I. Zlatev, Phys. Rev. D 55, 7701 (1997).

[27] A. Jahan, Braz. J. Phys. 37, 144 (2007).

[28] R. Rekioua and T. Boudjedaa, Eur. Phys. J. C 49, 1091 (2007).

[29] B. Mirza and M. Mohadesi, Theor. Phys. 42, 664 (2004).

[30] T. Curtright, D. Fairlie, and C. Zachos, Phys. Rev. D 58, 025002 (1998). 
[31] G. Magro, arXiv:quant-ph/0302001.

[32] J. Schwinger, Phys. Rev. 82, 664 (1951).

[33] C. Grosche and F. Steiner, Handbook of Feynman Path Integrals, Springer Tracts in Modern Physics, Vol. 145 Springer, Berlin, Heidelberg 1998.

[34] M. Moshinsky and A. Szczepaniak, J. Phys. A 22, L817 (1989).

[35] I. S. Gradshteyn and I. M. Ryzhik, Table of Integrals, Series, and Products, Academic Press, NewYork 1979.
[36] A. Smailagic and E. Spalucci, J. Phys. A 36, 467 (2003).

[37] S. Gangopadhyay and F. G. Scholtz, Phys. Rev. Lett. 102, 241602 (2009).

[38] V. M. Villalba and R. Maggiolo, Eur. Phys. J. B 22, 31 (2001).

[39] V. M. Villalba, Phys. Rev. A 49, 586 (1994). 